\title{
Expression of vascular endothelial growth factor in human oral squamous cell carcinoma: its association with tumour progression and p53 gene status
}

\author{
T Maeda, S Matsumura, H Hiranuma, A Jikko, S Furukawa, T Ishida, H Fuchihata
}

\begin{abstract}
Aims-To correlate vascular endothelial growth factor (VEGF) expression in oral squamous cell carcinoma with the clinicopathological characteristics and prognosis; and to assess whether p53 gene status is associated with VEGF expression in human cancers.

Methods-Tumour specimens from 45 patients with oral squamous cell carcinomas were examined. Expression of VEGF was determined using an immunohistochemical method, and a tumour was considered positive when more than $5 \%$ of the neoplastic cells showed VEGF immunoreactivity. The p53 gene status was screened using a polymerase chain reaction-single strand conformation polymorphism analysis.

Results-VEGF positive staining was detected in $19(42.2 \%)$ of the 45 cases. VEGF immunoreactivity did not correlate with the histological degree of tumour differentiation, clinical stages, or lymph node metastasis. The patients with VEGF positive tumours had a significantly worse prognosis than those with VEGF negative tumours. The five year overall survival rate of the VEGF negative patients was $76.5 \%$, as compared with $48.8 \%$ for the VEGF positive patients. No significant association between VEGF expression and the p53 gene status of the tumours was found.

Conclusions-VEGF is a good prognostic indicator of the survival of patients with oral squamous cell carcinoma. The p53 gene status does not seem to be associated with VEGF expression in these cancers. (f Clin Pathol 1998;51:771-775)
\end{abstract}

Keywords: vascular endothelial growth factor; p53; oral squamous cell carcinoma

Angiogenesis is an essential step in tumour growth and metastasis. ${ }^{1}$ It involves the formation of new blood vessels from pre-existing vessels by a multistep mechanism. ${ }^{2}$ Angiogenesis properties are correlated with tumour aggressiveness, and intratumour microvessel density has been found to be an independent prognostic factor.

It is known that tumour cells can release diffusible angiogenic regulatory factors. ${ }^{4}$ Vascular endothelial growth factor (VEGF) is currently considered a leading candidate among the fac- tors causing tumour angiogenesis. VEGF, which was first found to affect angiogenesis in malignant gliomas, ${ }^{5}$ stimulates the proliferation of endothelial cells ${ }^{6}$ and has been identified in many different tumours, particularly in hypoxic areas. $^{7}$ The inhibition of VEGF signalling inhibits both tumour angiogenesis and the growth of experimental solid tumours. ${ }^{8} 9$

It is thought that VEGF protein expression promotes tumour metastasis in human solid tumours. ${ }^{10}$ However, there is disagreement on the importance of VEGF in determining metastatic behaviour. ${ }^{11-13}$ With regard to the prognostic value of VEGF, some studies have shown that the expression of VEGF is an independent prognostic factor in patients with breast cancer. ${ }^{14-16}$ However, except in the case of breast cancer, few studies on the association between VEGF expression and cancer prognosis have been published. ${ }^{11} 13$

Recent in vitro experimental studies have indicated that the p53 tumour suppressor gene is closely involved in the regulation of VEGF mRNA expression. ${ }^{17}{ }^{18}$ These reports prompted us to investigate whether the VEGF expression pattern varies according to the p53 gene status in human cancers of patients.

We evaluated 45 cases of oral squamous cell carcinoma for the expression of VEGF by immunohistochemistry, and screened them for mutations of the p53 gene by polymerase chain reaction-single strand conformation polymorphism (PCR-SSCP) analysis. We investigated the correlations between the expression of VEGF protein and various clinicopathological factors including prognosis. In addition, we analysed the relation between VEGF expression and p53 gene status in these tumours.

\section{Methods}

PATIENTS AND SAMPLES

We examined 45 formalin fixed, paraffin embedded tissue samples of oral squamous cell carcinoma obtained by biopsy from 45 patients. The patients were enrolled at the Department of Radiology, Osaka University Dental Hospital, between October 1989 and February 1994. They were 28 males and 17 females, ranging in age from 32 to 87 years, with an average age of 63.7 years. All patients were staged according to the tumour-nodemetastasis (TNM) classification of tumours (Union Internationale contre le Cancer (UICC), 1987). One patient had stage I, 18 had stage II, 13 had stage III, and 13 had stage IV tumours. All patients were fresh cases and
Accepted for publication 8 June 1998 


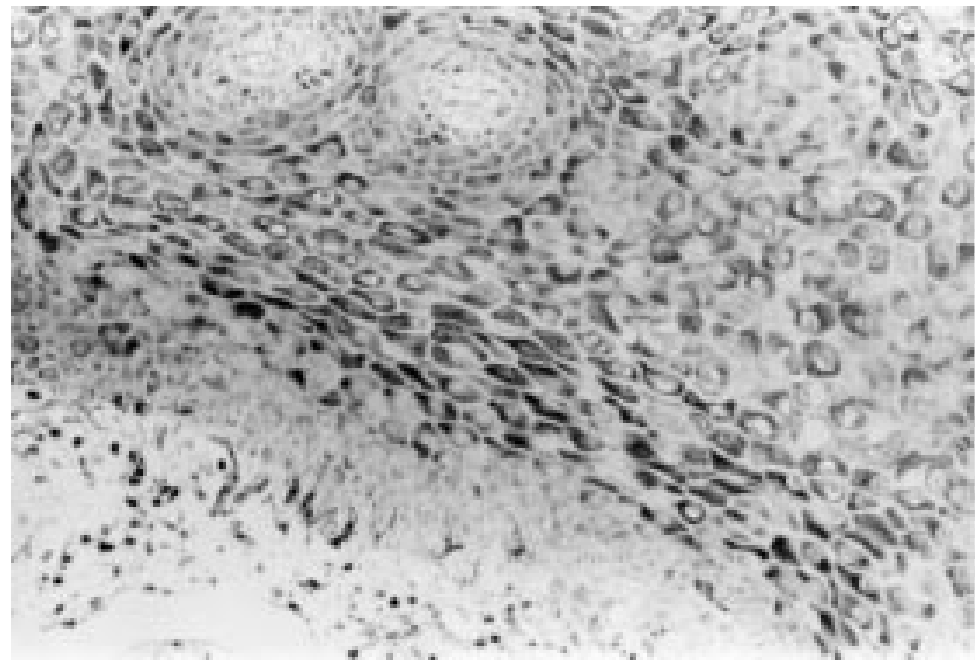

Figure 1 Representative examples of VEGF immunostaining in oral squamous cell carcinoma. There is a strong cytoplasmic staining of the tumour cells. (Magnification $\times 100$.)

had received no previous treatment. Of the 45 patients, 24 received radiotherapy alone and 21 received preoperative radiotherapy and then underwent surgery. Radiotherapy was provided by external beam megavoltage equipment, reaching a total dose of 50 to $70 \mathrm{~Gy}$. For the preoperative radiotherapy, a total dose of 20 to 40 Gy was given. The histological degree of tumour differentiation was determined from haematoxylin and eosin stained sections by the hospital's laboratory of clinical pathology.

VEGF IMMUNOSTAINING

The avidine-biotin complex method was used to detect VEGF immunoreactivity. In all cases, $5 \mu \mathrm{m}$ thick paraffin embedded sections were cut and mounted on silane coated glass slides. The sections were deparaffinised in xylene and rehydrated through a series of graded ethanol, and then incubated with $0.3 \%$ hydrogen peroxide in methanol for 30 minutes to inhibit endogenous peroxidase activity. The slides were placed in a $0.01 \mathrm{M}$ citrate buffer $(\mathrm{pH} 6)$ and heated in a microwave oven at $500 \mathrm{~W}$ for two periods of five minutes each. The sections were then washed in phosphate buffered saline (PBS) and incubated in $3 \%$ normal swine
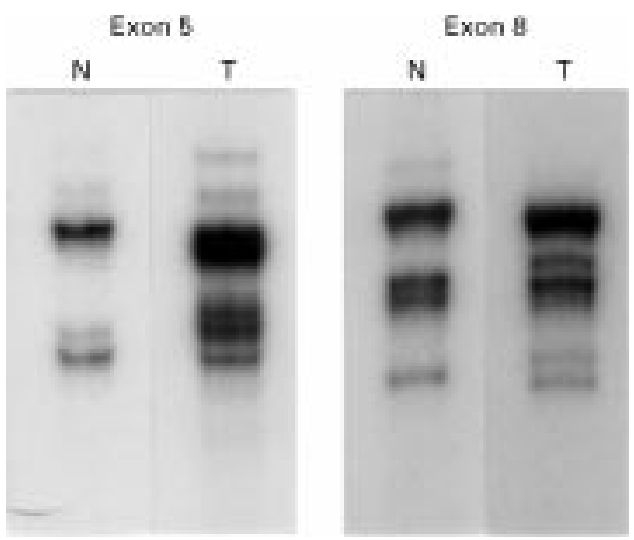

Figure 2 Typical examples of detection mutations by PCR-SSCP analysis in exons 5 and 8 of the p53 gene in oral squamous cell carcinoma (cases 21 and 37 , respectively). Compared with the normal control $(N)$ bands, abnormal band shifts can be seen in the tumours (T). serum for one hour to reduce non-specific antibody binding. As a primary antibody, A-20 (Santa Cruz Biotechnolog) was used. A-20 is a rabbit polyclonal antihuman VEGF antibody. The specimens were then incubated with a 1 : 200 dilution of A-20 overnight at $4^{\circ} \mathrm{C}$. After three washes with PBS, the sections were incubated with secondary biotinylated swine antirabbit antibody (Dako) at a dilution of 1: 500 for one hour. The sections were then exposed to avidine-biotin peroxidase complex (Vector Laboratories) for one hour. Finally, the reaction was made visible by incubation in $0.05 \mathrm{M}$ Tris- $\mathrm{HCl}$ buffer $(\mathrm{pH} 7.4$ ) containing $0.04 \%$ diaminobenzidine and $0.003 \%$ hydrogen peroxide with $0.1 \%$ nickel ammonium sulphate intensification. With each batch of staining runs, a positive control consisting of a tissue section from human normal kidney was used. In addition, negative controls were processed by omitting the primary antibody.

EVALUATION OF IMMUNOHISTOCHEMICAL

RESULTS

The results of the immunohistochemical staining were evaluated by two observers without prior knowledge of the patients' clinicopathological data. An individual tissue section was considered positive if unequivocal immunoreactivity was seen in more than $5 \%$ of the tumour cells, as previously described. ${ }^{11}$

DNA PREPARATION

The extraction of DNA from formalin fixed, paraffin embedded carcinoma tissues was performed by the proteinase $\mathrm{K}$ technique as previously described. ${ }^{19}$

PCR-SSCP ANALYSIS AND DIRECT SEQUENCING We screened p53 mutations in the 45 tumours from exon 5 to 8 using a PCR-SSCP analysis. The PCR was performed in a $50 \mu \mathrm{l}$ reaction mixture containing 100 ng genomic DNA, 20 pmol of each primer, $200 \mu \mathrm{M}$ each of dATP, dTTP, and dGTP, $20 \mu \mathrm{M}$ dCTP, $1 \mu \mathrm{Ci}$ $\left[{ }^{32} \mathrm{P}\right] \mathrm{dCTP}(3000 \mathrm{Ci} / \mathrm{mmol}), 10 \mathrm{mM}$ Tris$-\mathrm{HCl}$ (pH 8.3), $1.5 \mathrm{mM} \mathrm{MgCl}, 50 \mathrm{mM} \mathrm{KCl}$, and 2.5 units of Ampli-Taq polymerase (PerkinElmer). Thirty cycles of denaturation $\left(94^{\circ} \mathrm{C}\right.$, 30 seconds), annealing $\left(52-60^{\circ} \mathrm{C}\right.$, one minute), and extension $\left(72^{\circ} \mathrm{C}\right.$, two minutes) were carried out in a DNA thermal cycler (Perkin-Elmer). We used synthesised oligomer primers for exons 5 to 8 of the p53 gene. The primer sequences were: exon 5: 5'-TACTTCCCTGCCCTCAACAA-3' and 5'ATCGCTATCTGAGCAGCGCT-3'; exon 6: 5'-GATTGCTCTTAGGTCTGGCC-3' and 5'-CTGACAACCACCCTTAACCC-3'; exon 7: 5'-ACTGGCCTCATCTTGGGCCT-3' and 5'-TGTGCAGGGTGGCAAGTGGC-3'; exon 8: 5'-TAAATGGGACAGGTAGGACC-3' and 5'-TCCACCGCTTCTTGTCCTGC-3'. After the PCR, the reaction mixtures were diluted threefold with $0.1 \%$ sodium dodecyl sulphate (SDS) plus $10 \mathrm{mM}$ EDTA, and mixed with the same volume of loading dye solution. Following denaturation at $95^{\circ} \mathrm{C}$ for five minutes, the diluted samples were applied to $5 \%$ neutral polyacrylamide gels with $5 \%$ glycerol, and elec- 
Table 1 Relation between vascular endothelial growth factor (VEGF) expression and clinicopathological characteristics

\begin{tabular}{|c|c|c|c|}
\hline \multirow[b]{2}{*}{ Variable } & \multicolumn{2}{|l|}{$V E G F$} & \multirow[b]{2}{*}{$p$ Value } \\
\hline & Negative & Positive & \\
\hline \multicolumn{4}{|l|}{ Differentiation } \\
\hline Good & 10 & 4 & NS \\
\hline Moderate & 13 & 12 & \\
\hline Poor & 3 & 3 & \\
\hline \multicolumn{4}{|l|}{$\begin{array}{l}\text { Lymph node } \\
\text { metastasis }\end{array}$} \\
\hline Negative & 15 & 10 & NS \\
\hline Positive & 11 & 9 & \\
\hline \multicolumn{4}{|l|}{ Clinical stage } \\
\hline I & 1 & 0 & NS \\
\hline II & 12 & 6 & \\
\hline III & 6 & 7 & \\
\hline IV & 7 & 6 & \\
\hline \multicolumn{4}{|c|}{ Primary treatment } \\
\hline RT & 13 & 11 & NS \\
\hline RT + surgery & 13 & 8 & \\
\hline
\end{tabular}

RT, radiotherapy.

trophoresis was performed at a $40 \mathrm{~W}$ constant power for two hours at room temperature. The gel was dried on filter paper and exposed to $x$ ray film at $-80^{\circ} \mathrm{C}$. To confirm each mutations, DNAs were extracted from abnormal bands and directly sequenced using a dye terminator cycle sequencing FS kit (Applied Biosystems), according to the manufactures' instructions. Normal DNA samples from healthy human tissue were used as negative controls of band shifts.

STATISTICAL ANALYSIS

The relation between VEGF expression and the clinicopathological characteristics or $\mathrm{p} 53$ gene status was examined by the $\chi^{2}$ test. The Kaplan-Meier survival method ${ }^{20}$ was used for univariate analysis and the log rank test was used to assess differences between the two groups (VEGF positive and negative). Statistical significance was defined as $\mathrm{p}<0.05$. The StatView software (Abacus Concepts) was used for statistical analysis.

\section{Results}

VEGF IMMUNOSTAINING

The tumour specimens obtained from the 45 patients with oral squamous cell carcinoma were analysed immunohistologically for the expression of VEGF. VEGF immunoreactivity was mainly localised to the cytoplasm of the tumour cells (fig 1). Nineteen carcinomas $(42.2 \%)$ were VEGF positive and $26(57.8 \%)$ were negative.

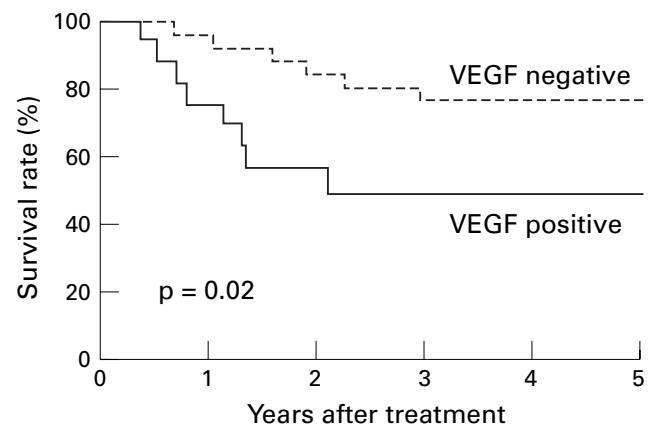

Figure 3 Kaplan-Meier curves for the overall survival of the patients according to the expression of vascular endothelial growth factor (VEGF). The two curves are significantly different.
Table 2 Vascular endothelial growth factor (VEGF) expression according to $p 53$ gene status

\begin{tabular}{llll}
\hline \multirow{2}{*}{ VEGF expression } & \multicolumn{2}{l}{$p 53$ Status } & \\
\cline { 2 - 3 } & Wild-type & Mutant type & p Value \\
\hline Negative & 18 & 8 & NS \\
Positive & 9 & 10 & \\
\hline
\end{tabular}

p53 GENE MUTATIONS

We identified p53 mutations in 18 (40.0\%) of the 45 tumour specimens. We found seven, two, four, and six mutational alterations in exons 5, 6, 7, and 8, respectively. One specimen had mutations in both exon 7 and 8. Typical examples of the PCR-SSCP of exons 5 and 8 are shown in fig 2 .

RELATION BETWEEN VEGF EXPRESSION AND CLINICOPATHOLOGICAL CHARACTERISTICS The correlations between VEGF expression and various clinicopathological characteristics are shown in table 1 . As for the primary treatment method, no bias was found between the VEGF positive and VEGF negative groups. There were no significant associations between VEGF expression and the histological degree of tumour differentiation or clinical stages.

Cervical lymph node metastasis was observed in 20 of the 45 patients at diagnosis. Eleven of the $26 \mathrm{VEGF}$ negative patients (42.3\%) developed lymph node metastasis, compared with nine of the $19 \mathrm{VEGF}$ positive patients $(47.4 \%)$. No significant correlation was found between VEGF expression and the incidence of lymph node metastasis.

The prognosis of the 45 patients was examined. We found it was worse in the patients with VEGF positive tumours than those with VEGF negative tumours. The overall survival rates of the VEGF negative and positive patients were significantly different $(\mathrm{p}=0.02)$. The five year overall survival rate of the VEGF negative patients was $76.5 \%$, compared with $48.8 \%$ for the VEGF positive patients (fig 3).

VEGF EXPRESSION ACCORDING TO p53 GENE STATUS

We analysed the relation between VEGF expression and p53 gene status in the 45 cases. Nine $(33.3 \%)$ of the 27 tumours without p53 gene mutations were VEGF positive, versus 10 (55.6\%) of the 18 tumours with p53 mutations (table 2). No significant correlation was found between the VEGF expression and p53 gene status, but there was a trend towards a positive relation between VEGF positivity and p53 mutations.

\section{Discussion}

Neovascularisation nourishes a growing tumour and also allows tumour cells to be in contact with the vascular bed of the host. Tumour angiogenesis is regulated by factors released either by the tumour cells themselves or by macrophages attracted into the tumour proper. ${ }^{21}$ Much work has been done in recent years to identify angiogenic factors. VEGF is the only angiogenic peptide known to act specifically on endothelial cells and is therefore considered to be the leading candidate of the 
factors playing a key role in tumour angiogenesis. ${ }^{22}$ Raised VEGF levels are frequently observed in tumour cells located near necrotic regions within the tumour, and it has been suggested that hypoxia may induce VEGF expression. ${ }^{7}$ In addition, Kim et al reported that tumour growth was suppressed by anti-VEGF treatment in vivo. ${ }^{8}$

VEGF expression has been found in a wide variety of tumour cells, and is detected mainly in the cytoplasm of the cells. In breast carcinomas $^{14}$ and gastric carcinomas, ${ }^{11}$ approximately half the tumour cells are VEGF positive. In the present study, 19 (42.2\%) of 45 oral squamous cell carcinomas showed cytoplasmic VEGF protein expression. There was no significant association between VEGF expression and the histological degree of tumour differentiation or the clinical stage.

The mechanism of metastasis is thought to be a complex of multiple steps including the degradation of extracellular matrix, the intravasation of tumour cells, and the attachment to distant organs. ${ }^{23}$ The role of VEGF in tumour metastasis remains unclear. An in vivo experimental study with VEGF transfected ovarian cells showed that VEGF did not make a tumour metastatic, ${ }^{24}$ whereas Claffey et al showed that VEGF transfection increased experimental metastasis of human melanoma cells. ${ }^{10}$ In a clinical study, Maeda et al reported a significant correlation between VEGF expression and lymph node metastasis or liver metastasis in patients with gastric carcinoma ${ }^{11}$ whereas no significant correlation was detected between VEGF expression and lymph node metastasis in lung carcinoma. ${ }^{13}$ In our present study, the expression of VEGF did not correlate with lymph node metastasis. VEGF may not influence lymphatic tumour metastasis.

We observed that VEGF expression in oral squamous cell carcinoma had prognostic significance. The prognosis of the patients with VEGF positive tumours was significantly worse than that of the patients with VEGF negative tumours. This is consistent with previous reports indicating that VEGF expression is an independent prognostic factor in patients with breast carcinoma, ${ }^{14}{ }^{15}$ lung carcinoma, ${ }^{13}$ or gastric carcinoma. ${ }^{11}$ These data suggest that the presence of VEGF expression may be a good prognostic indicator, along with other clinicopathological factors, in patients with oral squamous cell carcinoma.

Little is known about the regulation of VEGF expression. Hypoxia is thought to be one factor which promotes the expression of VEGF. There may well be other mechanisms of regulation for this important angiogenic protein. The tumour suppressor gene p53 encodes a nuclear phosphoprotein that is associated with cell cycle regulation, cellular differentiation, the suppression of abnormal cell proliferation, and tumour development. Since mutations of the p53 gene have been found in various types of human cancer, p53 has been considered a marker of both malignancy and tumour progression. ${ }^{25}$ The progression from low grade astrocytoma to the highly malignant glioblastoma involves the clonal expansion of p53 mutant cells. ${ }^{26}$ During the progression of these tumours towards high grade malignancy, an increase of neovascularisation has also been observed. ${ }^{26}$ This finding suggests a possible correlation between p53 mutations and enhanced production of VEGF. Recent in vitro studies have indicated that the p53 gene is closely involved in the regulation of VEGF. Kieser et al showed that the induction of VEGF $\mathrm{mRNA}$ and protein by activated protein kinase $\mathrm{C}$ was strongly synergistic with mutant but not wild-type p53 gene. ${ }^{17}$ However, Mukhopadhyay et al reported that wild-type p53 downregulated the endogenous VEGF mRNA level, whereas mutant forms of p53 had no effect. ${ }^{18}$

These observations prompted us to investigate whether there is a significant correlation between the VEGF expression and p53 gene status in human solid tumours. To our knowledge, this is the first such study in human patients. We screened the oral squamous cell carcinoma specimens for $\mathrm{p} 53$ gene status using a PCR-SSCP analysis instead of an immunohistochemical examination, because the immunohistochemical staining of p53 protein is not always dependent on p53 gene mutations. ${ }^{27}$ Of the 18 tumours with p53 gene mutations, 10 (55.6\%) showed VEGF positive staining, whereas of the 27 tumours without p53 gene mutations, nine (33.3\%) showed positive staining. Contrary to our expectation, we found no significant correlation between p53 gene status and VEGF expression. However, there was a trend towards a positive correlation between p53 mutations and VEGF expression. Further investigation is necessary to determine the effect of the p53 gene on VEGF expression. We believe that important information about the genetic regulation of angiogenesis in tumours will be acquired by more detailed in vitro and in vivo studies.

In conclusion, our present results indicate that the expression of VEGF may be a good prognostic indicator in patients with oral squamous cell carcinoma. The examination of VEGF expression before treatment may be useful in assessing the biological behaviour of such tumours and thereby suggesting the most effective treatment.

This study was partly supported by a grant-in-aid from the Ministry of Education, Culture and Science of Japan (No 07557367).

1 Folkman J. What is the evidence that tumours are angiogenesis dependent? F Natl Cancer Inst 1990;82:4-6.

2 Folkman J. Angiogenesis in cancer, vascular, rheumatoid and other diseases. Nature Med 1995;1:27-31.

3 Weidner N, Folkman J, Pozza F, et al. Tumor angiogenesis: a new significant and independent prognostic indicator in eary-stage breast carcinoma. I Natl Cancer Inst 1992;84: eary-stage

4 Weidner N. Intratumor microvessel density as a prognostic factor in cancer. Am f Pathol 1995;147:9-19.

5 Plate KH, Breier G, Weich HA, et al. Vascular endothelial growth factor is a potential tumor angiogenesis factor in human gliomas in vivo. Nature 1992;359:845-8.

6 Ferrara N. Vascular endothelial growth factor. Trends Cardiovasc Med 1993;3:244-50.

7 Shweiki D, Itin A, Soffer D, et al. Vascular endothelial growth factor induced by hypoxia may mediate hypoxiainitiated angiogenesis. Nature 1992;359:843-5.

$8 \mathrm{Kim} \mathrm{KJ}, \mathrm{Li} \mathrm{B}$, Winer J, et al. Inhibition of vascular endothelial growth factor-induced angiogenesis suppresses tumor lial growth factor-induced angiogenesis
growth in vivo. Nature 1993;362:841-4.

9 Millauer B, Shawver LK, Plate KH, et al. Glioblastoma growth inhibited in vivo by a dominant-negative Flk-1 mutant. Nature 1994;367:576-9. 
10 Claffey KP, Brown LF, del Aguila LF, et al. Expression of vascular permeability factor/vascular endothelial growth actor by melanoma cells increases tumor growth, angiogenesis, and experimental metastasis. Cancer Res 1996;56: $172-81$

11 Maeda K, Chung YS, Ogawa Y, et al. Prognostic value of vascular endothelial growth factor expression in gastric carcinoma. Cancer 1996;77:858-63.

12 Anan K, Morisaki T, Katano M, et al. Vascular endothelial growth factor and platelet-derived growth factor are potential angiogenic and metastatic factors in human breast cancer. Surgery 1996;119:333-9.

13 Volm M, Koomagi R, Mattern J. Prognostic value of vascular endothelial growth factor and its receptor Flt-1 in squamous cell lung cancer. Int $\mathcal{F}$ Cancer 1997;74:64-8.

14 Toi M, Hoshina S, Takayanagi T, et al. Association of vascular endothelial growth factor expression with tumor angiogenesis and with early relapse in primary breast cancer. $尹 p n$ genesis and with early relapse

15 Toi M, Inada K, Suzuki H, et al. Tumor angiogenesis in breast cancer: its importance as a prognostic indicator and
the association with vascular endothelial growth factor the association with vascular endothelial growth fact

16 Relf $M$, LeJeune S, Scott PA, et al. Expression of the angiogenic factors vascular endothelial cell growth factor, acidic and basic fibroblast growth factor, tumor growth factor beta-1, platelet-derived endothelial cell growth factor, placenta growth factor, and pleiotrophin in human primary breast cancer and its relation to angiogenesis. Cancer Res 1997;57:963-9.

17 Kieser A, Weich HA, Brandner G, et al. Mutant p53 potentiates protein kinase $C$ induction of vascular endothelial growth factor expression. Oncogene 1994;9:964-9.
18 Mukhopadhyay D, Tsiokas L, Sukhatme V. Wild-type p53 and v-Src opposing influence on human vascular endotheial growth factor gene expression. Cancer Res 1995;57: $6161-5$

19 Goeltz SE, Hamilton SR, Vogelstein B. Purification of DNA from formaldehyde fixed and paraffin embedded human tissue. Biochem Biophys Res Commun 1985;130:118-26.

20 Kaplan EL, Meier P. Non parametric estimation from incomplete observations. F Am Stat Assoc 1958;53:457-81.

21 Folkman J, Klagsbrun M. Angiogenic factors. Science 1987; 235:442-7.

22 Dvorak HF, Brown LF, Detmar M, et al. Vascular permeability factor/vascular endothelial growth factor, microvascular hyperpermeability, and angiogenesis. $\mathrm{Am} \mathcal{F}$ Pathol 1995;146:1029-39.

23 Liotta LA, Steeg PS, Stetler-Stevenson WG. Cancer metastasis and angiogenesis: an imbalance of positive and negative regulation. Cell 1991;64:327-36.

24 Ferrara N, Winer J, Burton T, et al. Expression of vascular endothelial growth factor does not promote transformation but confers a growth advantage in vivo to Chinese hamster cells. F Clin Invest 1993;91:160-70.

25 Levine AJ. The tumor suppressor genes. Annu Rev Biochem 1992;62:623-51.

26 Sidransky D, Mikkelsen T, Schwechheimer K, et al. Clonal expansion of p53 mutant cells is associated with brain tumor progression. Nature 1992;355:846-7.

27 Villuendas R, Piris MA, Algara P, et al. The expression of p53 protein in non-Hodgkin's lymphomas is not always dependent on p53 gene mutations. Blood 1993;82: 3151-6. 